\title{
Efecto de la motivación al logro y la inteligencia emocional en el Crecimiento Psicológico
}

\author{
Padrón López, Gladys Aracelys * \\ Sánchez de Gallardo, Marhilde **
}

\begin{abstract}
Resumen
Considerando como elementos claves de la excelencia gerencial, las características personales de los individuos que conforman las organizaciones, se efectuó un estudio aplicado, descriptivo, cuantitativo correlacional, observacional, de campo, con diseño no experimental transeccional correlacional-causal. El mismo presentó como objetivo general analizar el efecto de la motivación al logro y la inteligencia emocional en el crecimiento psicológico; todos ellos en personas exitosas en su ámbito de acción. Se diseñaron tres instrumentos tipo Lickert, validados a través del juicio de expertos, el poder discriminatorio de los ítems y la confiabilidad, estimada con el coeficiente Alpha Cronbach, arrojó para el de motivación al logro un $r_{\mathrm{tt}}=0.97$, inteligencia emocional $r_{\mathrm{tt}}=0.90$ y el de crecimiento psicológico $r_{t t}=0.97$. Los resultados procesados mediante estadísticas descriptivas e inferenciales, evidenciaron a las tres variables en un nivel muy alto, la relación entre la motivación al logro y la Inteligencia emocional con el Crecimiento Psicológico es alta y positiva ( $R$ de 0.820 ), explicando ambas el $67.3 \%$ de la variación total del Crecimiento Psicológico.
\end{abstract}

Palabras clave: Motivación al logro, inteligencia emocional, crecimiento psicológico.

Recibido: 10-06-09. Aceptado: 10-12-09

* Psicóloga, M.Sc. Administración de Empresas, Doctora Ciencias Gerenciales. Docente de pregrado y postgrado, Coordinadora de Trabajo Especial de Grado, Facultad de Ciencias Administrativas, URBE. E-mail: aracelyspl@hotmail.com

** Psicóloga. Mg. Orientación. Doctora Ciencias Gerenciales. Profesora Titular, Departamento de Psicología, Facultad de Humanidades y Educación, LUZ PPI, Nivel II. 0261-7596243.

E-mail: marsanchezg@cantv.net, marsanchezg@gmail.com. 
Motivación al logro e inteligencia emocional en el Crecimiento Psicológico

Padrón López, Gladys Aracelys y Sánchez de Gallardo, Marhilde

\title{
The Effect of Motivation on Achievement and Emotional Intelligence in Psychological Growth
}

\begin{abstract}
Considering that the personal characteristics of individuals that make up organizations are key elements for managerial excellence, an applied, descriptive study was conducted of the quantitative correlational, observational, field type, with a non-experimental, trans-sectional, correlational-causal design. The general objective was to analyze the effect of motivation on achievement and emotional intelligence in psychological growth for people who are successful in their areas of action. Three Lickerttype instruments were designed and validated by expert judgment; the discriminatory power of the items and their reliability, estimated with the Alpha Cronbach co-efficient, produced $r_{t t}=0.97$, for success motivation, $r_{\mathrm{tt}}=0.90$ for emotional intelligence and $r_{\mathrm{tt}}=0.97$ for pyschological growth. Results were processed using descriptive and inferential statistics, showing the three variables at a very high level. The relation between motivation for achievement and emotional intelligence with psychological growth is high and positive ( $R$ of 0.820 ), both expressing $67.3 \%$ of the total variation for psychological growth.
\end{abstract}

Key words: Motivation for achievement, emotional intelligence, psychological growth.

\section{Introducción}

Entre los aspectos fundamentales del desarrollo de las empresas y los países, se encuentra la excelencia de su talento humano. Al respecto, Chiavenato (2007) expone que individuos proactivos, dotados de inteligencia, creatividad, iniciativa, decisión, habilidades y competencias, dotados de una personalidad propia, con una historia personal y diferenciada, son indispensables para la adecuada administración de los recursos organizacionales. Romero (2000:1) hace referencia a "un alto nivel educacional, calidad profesional, entrenamiento técnico y científico" como la excelencia del talento humano de los ciudadanos de un país. En tal sentido, se requiere fomentar la velocidad de progreso de las personas, para el desarrollo de las empresas y naciones que conforman.

En este contexto, a medida que las organizaciones se desenvuelven en mer- cados más competitivos y dinámicos, producto entre otras cosas de la globalización, su entorno se vuelve cambiante. En el ambiente de incertidumbre en el cual se encuentran inmersas hoy en día, lo único constante es el cambio, y esto obliga a quienes las conducen a reflexionar e implantar diversos tipos de estrategias y acciones, a objeto de garantizar la competitividad organizacional, por cuanto ya no es suficiente sobrevivir, debe lograrse el éxito empresarial.

Debido a lo expuesto con anterioridad, los individuos que conforman las organizaciones deberán desarrollar sus características para adaptarse o mejor aun destacarse en este entorno cambiante, por cuanto sus sentimientos y habilidades son claves para el buen funcionamiento de las instituciones a las cuales pertenecen, $y$ de su vida en general. Bajo esta perspectiva, la presente investigación persigue como objetivo general analizar el efecto de la motivación al logro y la inteligencia emo- 
cional en el crecimiento psicológico; todos ellos en personas exitosas en su ámbito de acción y entre sus objetivos específicos: a) Analizar la dimensión interior y exterior de la motivación al logro. b) Establecer el perfil de competencias personales y sociales como elementos de la inteligencia emocional. c) Determinar el manejo positivo ante el fracaso, manejo de la incertidumbre, apertura al cambio y flexibilidad como elementos del crecimiento psicológico.

Este estudio se justifica considerando que dicho análisis proporcionará un acercamiento desde el punto de vista científico al conocimiento de la dinámica de variables internas de personas, lo cual podrá ser extrapolado a otros ámbitos tanto laborales como personales, brindado un patrón comparativo para aproximarse a la dinámica individual que garantice la excelencia del recurso humano y con ello al desarrollo de las organizaciones y los países.

A nivel organizacional, permitirá posteriormente modificar los subsistemas de ingresos, evaluación del desempeño, desarrollo, permitiendo detectar necesidades para diseñar e implementar acciones que permitan desarrollar a los individuos y así garantizar el recurso humano más apto y exitoso.

Los resultados generados se convertirán, por lo tanto, en una fuente de consulta para otros investigadores y gerentes, interesados en el estudio de las variables en referencia, por cuanto es innegable que el recurso humano en el activo mas importante, el único recurso dinámico de las organizaciones.

Así mismo, el conocimiento obtenido, podrá ser utilizado para organizar y promover cambios tanto individuales como grupales en el grupo objeto de estudio y gerentes de niveles similares.

En tal sentido, el ser humano constituye un factor clave para el éxito de los empresas, agrega valor real a las organizaciones, por lo cual debe tenerse información sobre dichos trabajadores en la ejecución de sus funciones, sobre sus fortalezas, debilidades y cualidades fundamentales relacionadas con la actuación exitosa en cualquier ámbito laboral.

La investigación puede ser clasificada como aplicada, de tipo descriptivo y cuantitativo correlacional, observacional y de campo. Asimismo, se utilizó un diseño no experimental transeccional correlacional-causal.

En cuanto a la población, considerando que el propósito del estudio y los criterios expuestos por Romero (2000:1), al referir que el desarrollo de los países proviene de la excelencia de su recurso humano y esto significa alto nivel educacional, calidad profesional, entrenamiento técnico y científico, se selecciona a los 165 doctorantes de gerencia y ciencias gerenciales de las universidades ubicadas en la ciudad de Maracaibo, lo cual lo delimita a la Universidad del Zulia y Rafael Belloso Chacín. Se utilizó el muestreo aleatorio estratificado proporcional, se estimó una muestra de 117 sujetos.

En dichos programas de postgrado, se agrupan profesionales de diversos tipos de instituciones, organizaciones y regiones del país, quienes como investigadores libres en su proceso de acreditación como doctores crean conocimientos y las innovaciones necesarias a los problemas gerenciales en su ámbito de acción. 
Motivación al logro e inteligencia emocional en el Crecimiento Psicológico Padrón López, Gladys Aracelys y Sánchez de Gallardo, Marhilde

Las observaciones realizadas parecen indicar que estos doctorantes por su manejo motivacional y emocional se constituyen en un modelo referencial de éxito, caracterizados por ser generadores de soluciones y de conocimiento, lo cual los caracteriza como personas altamente competitivas y exitosas.

En tal sentido, ellos comienzan sus estudios partiendo de su necesidad personal de desarrollo, y preocupación por el análisis de los diversos procesos gerenciales de sus organizaciones e instituciones, buscan generar propuestas de solución a los múltiples problemas por los que atraviesan las mismas ante los diversos cambios sociales políticos y económicos venezolanos, evidenciando de esta manera, su necesidad de ser proactivos y profundizar en los procesos de transformación y mejoramiento de la calidad y competitividad de las organizaciones a las cuales pertenecen.

Retomando planteamientos anteriormente referidos, dado que el desarrollo de un país depende de las personas, y la responsabilidad por el crecimiento personal es algo individual y responde a las demandas internas de los individuos, a su compromiso interior, a su forma de comportarse; este grupo de doctorantes se convierte en un grupo de interés analítico para la aproximación en el conocimiento de la estructura motivacional y emocional la cual parece caracterizar a las personas exitosas capaces de producir cambios en sí mismos y en las personas que los rodean.

Dentro de este marco, aproximarse a este conocimiento permitirá indagar sobre elementos y/o variables como la motivación al logro, inteligencia emocional y crecimiento psicológico, que podrían contribuir a delinear cual es la vía que las organizaciones y países en general deben transitar para el desarrollo de manera integral y poder enfrentar los cambios caracterizadores del mundo globalizado.

Por otra parte, la técnica seleccionada como fuente para la recolección de la información es la observación mediante encuesta, utilizando tres (3) cuestionarios, tipo escala Likert. El de motivación al logro conformado por veinticuatro (24) reactivos, los cuales reflejan las dimensiones interior (intrapersonal) y exterior (interpersonal); y cinco factores que miden diferentes componentes de logro: a) experticia, b) eficiencia, c) excelencia, d) crecimiento de otras personas, y e) calidad de vida social. El de inteligencia emocional con sesenta y dos (62) con las dimensiones competencia personal y competencia social y sus diecinueve indicadores; y el de crecimiento psicológico con quince (15) ítems, para los cuatro elementos: a) manejo positivo del fracaso; b) manejo de la incertidumbre; c) apertura al cambio, y d) flexibilidad.

Los mismos fueron validados a través del juicio de expertos, el poder discriminatorio de los ítems, utilizando la prueba t de Student; y la confiabilidad fue estimada con el coeficiente Alpha Cronbach, arrojando para el instrumento de motivación al logro un $r_{t t}=0.97$, inteligencia emocional con un $r_{\mathrm{tt}}=0.90$, y para el de crecimiento psicológico el $r_{\mathrm{tt}}=0.97$, lo cual indica la alta consistencia, seguridad y equilibrio de los cuestionarios.

En cuanto a la muestra, se procedió a determinar su tamaño, para lo que se utilizó la fórmula para universos finitos expuesta por Sierra Bravo (1999), la cual despejando la fórmula sobre la base del 
tamaño de la población, se obtiene que el tamaño ideal de la muestra es 117. Por tanto, la muestra quedó conformada para efectos del análisis por 117 doctorantes de gerencia y ciencias gerenciales de las universidades del Zulia y Rafael Belloso Chacín ubicadas en la ciudad de Maracaibo.

Una vez estimado el tamaño de la muestra, se determinó quienes formarán parte de ésta, para tal efecto se recurrió al muestreo aleatorio estratificado, dado que la población se encuentra clasificada por universidad. Considerando que los grupos no son del mismo tamaño, se seleccionó el estratificado con afijación proporcional, quedando conformada por Universidad LUZ: Doctorado en Gerencia, muestra 21 doctorantes, Universidad URBE: Doctorado de Ciencias Gerenciales 61 sujetos y Doctorado en Ciencias Mención Gerencia 35.

Para la selección de los ciento diez y siete (117) sujetos que conformaron la muestra objeto de estudio, se consideró pertinente la utilización de un muestreo probabilístico al azar simple y se utilizó la selección de los sujetos utilizando una tabla de números aleatorios, partiendo de un listado donde cada integrante de la población fue numerado, de tal manera que los resultados puedan ser generalizados al resto de la población objeto de estudio.

Para llevar a cabo el análisis de los datos obtenidos con los instrumentos, se utilizaron las estadísticas descriptivas frecuencias absolutas ( $\mathrm{fa}$ ) y porcentajes (\%), medidas de tendencia central y de dispersión, con los correspondientes baremos de interpretación, así como un análisis de regresión lineal múltiple.

\section{Motivación al logro. Algunas consideraciones}

Es innegable, ante el fenómeno globalizador que caracteriza el mundo actual, el desarrollo tecnológico, el cambio constante y el intenso movimiento en búsqueda de la calidad y la productividad, que las organizaciones se encuentran inmersas hoy en día en un ambiente de imprevisibilidad e incertidumbre; en este contexto varios factores han contribuido a este fenómeno, entre estos pueden ser mencionados: los cambios económicos, tecnológicos, sociales, culturales, jurídicos, políticos y demográficos.

En función de esta situación, las organizaciones en Venezuela han orientado sus esfuerzos a innovar y crear nuevos mecanismos para mantenerse y ampliar las posiciones ganadas en sus respectivas actividades, por cuanto en este entorno cambiante e imprevisible, según Dessler (2009) con tendencias hacia la globalización, modificaciones en la naturaleza del trabajo y la tecnología, afirma Hamel (referido por Gómez, 2003:6) "Es máas difícil que nunca mantener el éxito".

El objetivo es abrir nuevos horizontes con miras a enfrentar las exigencias de un mundo global, considerando que la realidad varía aceleradamente y para aprovechar los cambios en las organizaciones, señala Gómez (2003:7), nunca ha sido más válido el conocimiento gerencial, razón por la cual se ha denominado a esta época, la era del conocimiento.

Planteamiento reforzado por Ivancevich, Konopaske y Matteson (2006) al señalar la importancia de la flexibilidad y la capacidad de adaptación ante los cambios externos e internos, el comporta- 
Motivación al logro e inteligencia emocional en el Crecimiento Psicológico Padrón López, Gladys Aracelys y Sánchez de Gallardo, Marhilde

miento innovador y el desafío de riesgos, donde se apoya la perspectiva de la relevancia de las personas en las organizaciones que conforman.

En relación con las ideas expuestas, Chiavenato (2002:XXIII) señala que en este contexto globalizador, de desarrollo y cambio, la diferencia, la principal ventaja competitiva de las empresas se deriva de las personas. Según sus planteamientos, son las personas quienes generan y fortalecen la innovación y el futuro de las organizaciones.

Desde su perspectiva, la comparación entre países se realiza en términos de crecimiento psicológico, proceso referido por Romero (2000) como aquel mediante el cual el individuo enriquece la percepción de sí mismo y de su entorno, mejorando su relación con ambos, lo cual ocurre cuando desarrolla sus potencialidades, usa a plenitud sus capacidades y destrezas para beneficio propio y colectivo.

El crecimiento psicológico, destaca Romero (2000:2), requerido para impulsar el desarrollo de las naciones “... debe ser integral: interior y exterior, espiritual y material, para un país y para todos lo países". De lo cual se evidencia que para fortalecer un país se necesita incrementar el desarrollo de las personas, y el medio para hacerlo es la educación en cualquiera de sus formas, considerándola como un proceso espontáneo (informal) e institucional (formal) dirigido a propiciar el crecimiento psicológico. Continúa el autor, señalando que no pueden concebirse países tecnológica y científicamente desarrollados habitados por personas psicológicamente subdesarrolladas.

Adicionalmente debe considerarse que en lo fundamental, las organizacio- nes están constituidas por conjuntos de personas. Al hablar de las organizaciones, es necesario mencionar a quienes las representan, vivifican y les dan personalidad propia, es decir, de su talento humano.

Todo lo expuesto hasta el momento permite hacer ciertas reflexiones. Si se considera el contexto globalizador existente, los cambios frecuentes y dinámicos (lo cual podría dificultar la posibilidad de tener éxito), el hecho que la diferencia entre países se centre en su recurso humano, por cuanto es el activo dinámico y mas importante, pareciese indicar la dirección de los esfuerzos a realizar para garantizar la supervivencia y éxito de las organizaciones, estos deberían enfocarse en el desarrollo y/o fortalecimiento de quienes la conforman, los seres humanos.

En concordancia con lo expuesto, la realidad indica que las personas, cada día, se ven obligadas a aprender nuevas habilidades, oficios y profesiones, perfeccionarse y actualizarse. La presión social, tal como refiere Romero (2000:73), para el crecimiento ocupacional será mayor en las sociedades industriales orientadas hacia mercados globales, donde se necesita ser cada vez más productivo y competitivo, por lo cual: ... los países están obligados a entrenar a su fuerza laboral en las actividades requeridas por los nuevos procesos de producción. Además, los avances en la informática y las telecomunicaciones tienen como efecto secundario indeseable la eliminación de puestos de trabajo.

Lo anteriormente referido, está indicando que las personas deben desarrollar al máximo sus competencias ocupa- 
cionales y personales para hacerse competitivos e incrementar sus probabilidades de conseguir empleo, esto exige, tal como indica Romero (2000:74), cultivar las capacidades y destrezas instrumentales para el desarrollo de las personas.

Lo señalado implica crecer psicológicamente, desarrollar cualidades personales particulares, las cuales entre otros elementos, se relacionan con las motivaciones sociales, y la inteligencia emocional; lo cual se evidencia en los indicadores de crecimiento psicológico señalados por el autor: apertura y flexibilidad ante nuevas experiencias, manejo eficiente de situaciones de crisis y aprendizaje ante los fracasos, todos tienen tal como indica Romero (2000:94) “... un piso en común: La presencia del desafío, del reto para la persona que enfrenta la situación".

Entre estos elementos particulares, haciendo referencia especifica a las motivaciones sociales, debe señalarse que los hallazgos encontrados en diversos estudios reseñados por Romero (2000:102), permiten afirmar: “... la motivación al logro es la motivación para el crecimiento psicológico. Las personas con alta motivación al logro pueden crecer y crecen psicológicamente más que las otras."

Motivación al logro

Newstrom (2007) concibe la motivación al logro como un impulso que estimula a algunas personas para que persigan y alcancen sus metas. Un individuo con este impulso desea alcanzar objetivos y ascender por la escalera del éxito; siendo el logro visto como algo importante principalmente por sí mismo, no sólo por la recompensas que lo acompañan.
Feldman (2006) la conceptualiza como una característica aprendida y estable en la que la persona obtiene satisfacción al esforzarse por alcanzar un nivel de excelencia y conseguirlo.

Según Morris y Maisto (2005) las personas con motivación alta hacia el logro tienen una esperanza más fuerte de tener éxito que su temor al fracaso, son corredores de riesgos moderados más que altos o bajos y persisten en el esfuerzo cuando las tareas se vuelven difíciles.

Robbins y Coulter (2005) exponen como características de las personas con alto impulso de logro: Luchan por obtener logros personales más que por símbolos y recompensas del éxito, tienen el deseo de hacer algo mejor o de manera más eficiente que como se ha hecho antes, prefieren trabajos que ofrecen responsabilidad personal para encontrar soluciones a problemas, en los que puedan recibir una retroalimentación rápida e inequívoca sobre su desempeño con el propósito de saber si están mejorando y en los que puedan establecer objetivos moderadamente desafiantes; no son jugadores, ya que no les gusta lograr el éxito por casualidad, prefieren el reto de trabajar con un problema y aceptar la responsabilidad personal del éxito o el fracaso.

Ríos (2008) presenta como principales características del comportamiento típico de la motivación al logro (adaptados de Fernández-Abascal, Martín y Domínguez, 2001): Búsqueda activa del éxito en el rendimiento profesional, asunción de riesgos, aunque sin exceder capacidades reales; conductas emprendedoras, con interés en los negocios o actividades empresariales; se responsabilizan por 
Motivación al logro e inteligencia emocional en el Crecimiento Psicológico Padrón López, Gladys Aracelys y Sánchez de Gallardo, Marhilde

sus propios comportamientos; exponen interés por la información sobre su desempeño; búsqueda activa de nuevas formas de realizar las tareas que conduzcan al objetivo deseado; ejecución más eficaz en las tareas que les resulten desafiantes; mejor rendimiento ante tareas que suponen motivación intrínseca; preferencia por tareas de dificultad moderada; se evitan riesgos extremos, aunque asumiendo muchos riesgos calculados.

Santrock (2006) refiere a Mc. CleIland, 1985; Thrash y Elliot, 2002, quienes argumentan que una gran necesidad de logro por lo general produce resultados positivos, al menos en una sociedad orientada al éxito.

Complementariamente, la motivación al logro fue definida considerando los planteamientos de Romero (2000:41) como "... una red de conexiones cognitivo-afectivas relacionadas con el desarrollo personal, implicando un uso exigente de capacidades y destrezas para el beneficio personal y colectivo".

Evidenciándose de esta definición psicosocial que las interconexiones entre pensamientos, afectos y conducta en áreas sociales específicas, motivan todo el funcionamiento de la persona, tanto en lo interior (psíquico) como en lo exterior (social), dinamizando, orientando y manteniendo la conducta hacia metas interiores y exteriores valorizadas por la persona; para abordarla debe entonces, considerarse el análisis de sus dos dimensiones: dimensión interior o intrapersonal y la exterior o interpersonal. La primera, según Romero (2000:31), conformada por la experticia, referida a conocimientos profundos sobre tópicos o haceres específicos, eficiencia, definida como el uso productivo de habilidades, recursos y tiempo, y excelencia, descrita como resultados óptimos y la segunda, constituida por el impacto sobre el crecimiento de otras personas y la calidad de vida social. Considerando su definición, se clarifica que por lo general se piensa en la motivación al logro, siempre volcada hacia el interior y las personas con alta necesidad de logro únicamente interesadas en el propio desarrollo o éxito, lo cual no es cierto a la luz de la siguiente reflexión. Más allá de la entrega al trabajo y el placer generado por la propia ejecución, los sujetos con alta motivación al logro, sienten la necesidad de conocer el impacto de los resultados sobre familiares, compañeros de trabajo, comunidad y nación. Evidenciando de esta manera, una integración de lo individual y colectivo en la obra de los grandes hacedores de ciencia, arte, tecnología o productos industriales.

Bajo esta perspectiva se adiciona otro elemento de suma importancia, en el desarrollo personal y organizacional: la inteligencia emocional.

\section{Inteligencia emocional. Reflexiones teóricas}

Según Goleman (2000:385) dicha variables se refiere a la capacidad del individuo de reconocer sus propios sentimientos y los ajenos, de motivarse, manejar sus emociones y en las relaciones que establece con los demás.

Para DuBrin (2008) la inteligencia emocional se refiere a cualidades como entender los propios sentimientos, tener empatía con los demás y regular las propias emociones, para mejorar la forma de vivir. Una persona con gran inteligencia 
emocional podrá anticipar en comportamientos como evaluar a la gente, complacer a los demás e influir en ellos.

Este autor enuncia cuatro factores claves incluidos en un análisis reciente sobre la inteligencia emocional, expuestos por Goleman, Boyatzis y Mc-Kee (2004): Conocimiento de uno mismo, autocontrol, sensibilidad social y manejo de las relaciones.

El conocimiento de uno mismo se define como la capacidad de entender su humor, emociones y necesidades, así como también el impacto que éstas tienen sobre los demás. También incluye el uso de la intuición para tomar decisiones con las que se puede vivir feliz.

El autocontrol se refiere a la capacidad para controlar las propias emociones y actuar con honestidad e integridad de una forma constante y aceptable.

La sensibilidad social incluye la empatía con los demás y la intuición acerca de los problemas. También la señalan como otra faceta de la habilidad de interpretar la comunicación no verbal.

Describe el manejo de las relaciones como las habilidades interpersonales de poder comunicarse de manera clara y convincente, resolver los conflictos y construir fuertes lazos personales.

Así la inteligencia emocional, incorpora muchas de las habilidades y actitudes necesarias para lograr relaciones interpersonales eficientes en las organizaciones.

Matthews, Zeidner y Robert, 2003; Meyer et al, 2003, (citados por Feldman, 2006) describen la inteligencia emocional como el conjunto de habilidades que sustentan la valoración, evaluación, expresión y regulación precisa de las emociones.
Goleman (1995) (citado por Feldman, 2006) señala que la inteligencia emocional impulsa la capacidad para llevarse bien con los demás, permite comprender lo que se siente y experimenta y responder apropiadamente a las necesidades, considerando la inteligencia emocional como el fundamento de la empatía hacia los demás, la conciencia personal y las habilidades sociales. Destaca que una marcada inteligencia emocional permite que un individuo se sintonice con los sentimientos de los demás, lo que posibilita un elevado grado de sensibilidad hacia ellos.

Goleman, citado por Larsen y Buss (2005) concluyó que las medidas tradicionales de inteligencia, aunque predicen bastante bien el rendimiento escolar, en realidad hacen un trabajo pobre para predecir resultados posteriores como el logro ocupacional, salario, posición profesional. Afirma Goleman que la inteligencia emocional es más predictiva en forma sólida de estos resultados de la vida.

En este orden de ideas, en la actualidad no sólo se juzga a los individuos por su inteligencia, formación o experiencia, sino también por el modo en el cual se relacionan consigo mismos o con los demás. Por tal razón, desde todos los ámbitos se considera la inteligencia emocional como clave para el éxito personal y profesional. Para las organizaciones, conseguir al más brillante en algún ámbito es cuestión de tiempo y dinero, pero será mucho más complicado encontrar al más apto.

Lo anterior significa inteligencia emocional. Esta última constituye un soporte fundamental de la eficacia de la actuación gerencial y aporta guías del mayor interés para que el ejercicio de la dirección colabore sucesivamente en la 
Motivación al logro e inteligencia emocional en el Crecimiento Psicológico Padrón López, Gladys Aracelys y Sánchez de Gallardo, Marhilde

mejora progresiva de la capacidad de liderazgo.

La acción emocionalmente inteligente es trascendental por dos motivos: primero, porque es la base de los comportamientos eficaces, y por lo tanto, también de los resultados que se obtienen, y segundo, porque ocasiona la repercusión indirecta o colateral, pero de gran importancia, de afianzar positiva y constructivamente la experiencia adquirida como gerente $y$, por ello, también mejora la aptitud directiva de cara a sus actuaciones futuras.

Dichos planteamientos se confirman con lo expuesto por Cooper y Sawaf (1998:XIII) al señalar que la inteligencia emocional sustenta "... muchas de las mejores decisiones, las organizaciones más dinámicas y rentables, y las vidas más satisfactorias y de éxito."

De este modo, resulta ciertamente paradójico que las habilidades "blandas" relacionadas con las competencias emocionales, en contraposición con los dominios más duros representados por las capacidades cognitivas tengan una importancia decisiva en el éxito profesional, la excelencia depende más de las primeras. Incluso en las profesiones técnicas $y$ científicas, el pensamiento analítico ocupa un tercer lugar, después de la capacidad de influir sobre los demás y de la motivación de logro. El objetivo esencial lo constituye entonces, el mejoramiento de la productividad del talento humano en pro del mejoramiento de las empresas haciéndolas más eficientes y eficaces.

Relacionado con lo anterior, Goleman, Boyatzis y McKee (2004:309), formalizaron un modelo actualizado para su estudio, en función de datos recopilados en investigaciones y de los consiguientes análisis estadísticos, el mismo tiene cuatro dominios (conciencia de sí mismo, autogestión, conciencia social y gestión de las relaciones) y diecinueve competencias respectivamente.

En este contexto debe señalarse que los estudios realizados por Goleman (referidos por Romero, 2000:151) proporcionan apoyo indirecto al planteamiento sobre la importancia del manejo motivacional y emocional para producir cambios en el propio individuo y en las personas con las cuales se relaciona, en concordancia con lo cual expresa: Para cambiarnos y cambiar a otros en dirección de un verdadero crecimiento, lo ideal es fundir en un solo acto las ideas inteligentes y oportunas con el manejo motivacional-emocional que generen en nuestra audiencia una identificación razonada y entusiasta.

Extrapolando dichas ideas a los ambientes organizacionales, es posible afirmar que el secreto del éxito, es la gente, aquellos quienes la conforma, en tal sentido para lograrlo no son suficientes los conocimientos adquiridos en la formación universitaria, porque en la era de la información, el poder no lo posee quien conoce, sino quien continua aprendiendo, las personas que se desarrollan para encarar y desempeñarse efectivamente en ambientes de permanentes cambios, por cuanto se requieren nuevas habilidades para competir.

\section{Crecimiento psicológico. Algunas reflexiones}

Esta variable ha sido definida por Romero (2000:75) como “... un proceso a través del cual la persona genera cons- 
trucciones integradoras cada vez más complejas sobre su realidad interior (individual) y exterior (social) que le significan cambios positivos como ser humano".

Lo anteriormente referido estaría indicando que se manifiesta en dos perspectivas, interior y exteriormente, considerando al ser humano como persona indivisible, este enfoque psicosocial exige la expresión del crecimiento en el interior de la persona y en el entorno social en el cual se desenvuelve.

En el modelo propuesto por el autor para su estudio, se contemplan indicadores que tienen como base común, “... La presencia del desafío, del reto para la persona que enfrenta la situación" (p. 94).

Desde la perspectiva de Romero (2000:94) el manejo positivo del fracaso fue definido como la capacidad para utilizar la información presente en la situación de fracaso de manera favorable para el crecimiento personal, esto implica reinterpretar una realidad y esforzarse por alcanzar una meta; y el manejo de la incertidumbre como la capacidad para construir las situaciones inciertas o impredecibles de manera favorable para el propio crecimiento. Dichas situaciones se convierten entonces, en retos y oportunidades, contienen un potencial liberador de los limites del ser humano.

Por tanto, las situaciones de fracaso que enfrenta el ser humano se constituyen como oportunidades para aprender y crecer, aumentan las fortalezas y agudizan los sentidos, considerando la oportunidad de aprendizaje para generar construcciones y probar las habilidades y persistencia conductual; y desde la óptica de Gordon (2000:422) el manejo de la incertidumbre, tiene relación con la habilidad del individuo para enfrentar y compensar los entornos cambiantes.

En tal sentido, de acuerdo a los planteamientos de Reeve (2003) el manejo positivo del fracaso, podría incrementar las cualidades de competencia que involucran la necesidad del desafío óptimo. Además, al manejar adecuadamente la incertidumbre, según lo expuesto por Robbins (2004:65), se sentirán más o menos cómodos ante los riesgos, tolerando, relativamente los comportamientos y opiniones de otros, por cuanto no los percibirán como amenazas.

Aperturarse al cambio, tal como indico Romero (2000:99), hace referencia a la disposición de la persona a exponerse a experiencias nuevas y crecer a partir de ellas, atreviéndose a hacer algo nunca hecho, lo anterior implica exposición a obtener cualquier tipo de resultados. En tal sentido, significa tal como indica Mittelman (1991; referido por Reeve, 2003), una forma de recibir información y sentimientos de modo que ninguno se reprima, ignore, filtre o distorsione por deseos, temores o experiencias pasadas.

El cambio de acuerdo a lo expuesto por French, Bell y Zawacki (2007), debe adoptarse desde una perspectiva que lo considere como una oportunidad, no como una amenaza, tomando en cuenta que tanto las demandas de cambio provenientes del exterior de las organizaciones, tales como, dependencias gubernamentales, competidores, nuevas tecnologías, clientes, así como las que se derivan del interior de las mismas, como productos o servicios obsoletos, una nueva dirección estratégica, son constantes, por lo consiguiente resulta necesario comprenderlo como una fuerza siempre 
Motivación al logro e inteligencia emocional en el Crecimiento Psicológico Padrón López, Gladys Aracelys y Sánchez de Gallardo, Marhilde

presente y no percibirlo como un obstáculo, indeseable y perturbador.

Los planteamientos anteriores guardan relación con los aportes proporcionados por Sánchez de Gallardo y Linares (2006) cuando exponen que en la actualidad se asume al gerente dentro de un enfoque integral en el cual se combinan sus capacidades para dirigir, organizar, planificar y controlar la gestión de la organización con sus atributos personales, entre los que se incluyen: su liderazgo, su habilidad comunicativa, su capacidad para tomar decisiones, su motivación, su poder de convencimiento y de convocatoria, su habilidad para conformar equipos efectivos de trabajo, su autoestima, entre otros.

Consideran el enfoque de capital humano, con una premisa fundamental la potencialización y aprovechamiento de las condiciones personales de los individuos, destacando su importancia y su activa incidencia en el logro de los objetivos organizacionales. En este sentido, dichos elementos personales, son considerados como el principal activo de las organizaciones, están implicados en la gestión institucional y vitales para la consecución de los fines hacia los cuales las empresas e instituciones, de diversa índole, dirigen sus esfuerzos.

A continuación se exponen los resultados generados de esta investigación referidos a variables personales mencionadas anteriormente.

\section{Resultados}

Efecto de la Motivación al Logro e Inteligencia Emocional en el Crecimiento Psicológico

Los estadísticos inferenciales estimados permiten afirmar la existencia de una relación entre la motivación al logro y la inteligencia emocional con el crecimiento psicológico alta y positiva $(R$ de 0.820 ) y revelar que el $67.3 \%$ de la variación total del crecimiento psicológico es explicada por la motivación al logro y la inteligencia emocional $\left(R^{2}\right.$ de 0.673$)$ en el grupo objeto de estudio.

Lo anteriormente referido se confirmó con el análisis de la varianza estimado, señalando la existencia de un adecuado ajuste del modelo lineal de los datos ( $F$ de 117.079), es decir, el crecimiento psicólogo es explicado por la combinación lineal de la motivación al logro y la inteligencia emocional, indicando una contribución mayor para la explicación del modelo lineal múltiple de la motivación al logro (coeficiente $B$ de 0.431) sobre la inteligencia emocional (coeficiente B de 0.420).

Se encontró que las tres variables se ubicaron en el grupo en un nivel muy alto, la motivación al logro (94.9\%) con una baja dispersión de los puntajes indicó poca variabilidad en su comportamiento; mientras el comportamiento de la inteligencia emocional $(89.7 \%)$ y el crecimiento psicológico $(88 \%)$ con una moderada dispersión, mostró el predominio de los sujetos en el nivel en referencia aun cuando se ubicaron entre un alto a muy alto nivel en cada una de las variables referidas (Tabla 1).

Motivación al Logro: Análisis de la dimensión interior y exterior

En cuanto a la dimensión interior o intrapersonal, los resultados obtenidos en los tres indicadores que la conforman, evidenciaron un comportamiento similar por cuanto la experticia $(95.7 \%)$, eficiencia $(94.9 \%)$ y excelencia $(94 \%)$ en el grupo objeto de estudio, los cuales se ubica- 
Tabla 1

Estadísticos descriptivos e inferenciales: Motivación al logro, inteligencia emocional y Crecimiento Psicológico

\begin{tabular}{|c|c|c|c|}
\hline Variable & Porcentaje & Interpretación & Dispersión \\
\hline Motivación al Logro & $94.9 \%$ & Muy Alta & Baja (15.73) \\
\hline Inteligencia Emocional & $89.7 \%$ & Muy Alta & Moderada (36.81) \\
\hline Crecimiento Psicológico & $88 \%$ & Muy Alta & Moderada (10.69) \\
\hline Regresión Lineal Múltiple & \multicolumn{3}{|c|}{$\begin{array}{c}\text { Coeficiente de correlación múltiple } 0.820(\mathrm{a}) \\
\text { Coeficiente de determinación } \mathrm{R}^{\wedge} 2 \quad 0.673\end{array}$} \\
\hline Análisis de la Varianza (b) & \multicolumn{3}{|c|}{ F 117.079} \\
\hline $\begin{array}{l}\text { Modelo de Regresión } \\
\text { Lineal Múltiple } \\
\text { Variables en el Modelo }\end{array}$ & $\begin{array}{r}\text { Co } \\
\text { Motivació } \\
\text { Inteligencia }\end{array}$ & $\begin{array}{l}\text { les } \\
\text { nte } \\
\text { ogro (ML) } \\
\text { ocional (IE) }\end{array}$ & $\begin{array}{c}\text { Coeficientes } \\
\text { estandarizados } \\
\text { Beta (B) } \\
1.121 \\
0.431 \\
0.420\end{array}$ \\
\hline
\end{tabular}

Fuente: Padrón y Sánchez de Gallardo (2009).

(a) Variables predictoras: (Constante), Inteligencia emocional, Motivación al Logro.

(b) Variable dependiente: Crecimiento psicológico.

ron en un nivel muy alto, con una baja dispersión. En concordancia, la dimensión interior se situó en un nivel muy alto $(95.7 \%)$ con baja dispersión.

Continuando con el análisis de la dimensión exterior o interpersonal, debe señalarse que los resultados obtenidos demostraron un nivel muy alto en relación al impacto sobre el crecimiento de otras personas ( $88 \%$ ) y la calidad de vida social $(86.3 \%)$, aun cuando la dispersión encontrada en ambos casos fue moderada. En concordancia con lo anterior, la dimensión exterior o interpersonal se ubicó en un nivel muy alto $(89.7 \%)$, con una moderada dispersión.

Inteligencia Emocional: Perfil de Competencias Personales y Sociales

El perfil de inteligencia emocional que caracteriza al grupo objeto de estu- dio, como representantes de personas exitosas en su ámbito de acción, de acuerdo a los resultados obtenidos, demostró como la competencia personal $(89.7 \%)$ conformada por la conciencia de sí mismo y autogestión; y por otra parte la social $(89.7 \%)$ constituida por la conciencia social y la gestión de las relaciones, se encuentran ambas en un nivel muy alto.

En cuanto a los dominios utilizados para su estudio, en función del modelo teórico expuesto por Goleman, Boyatzis y McKee (2004) se evidenciaron niveles altos con dispersiones moderadas para la conciencia de sí mismo (76.9\%), autogestión (88.9\%) y conciencia social $(86.3 \%)$, indicando que la mayoría de los sujetos se distribuyeron entre niveles muy altos y altos; en referencia a la gestión de relaciones (93.2\%), también se 
Motivación al logro e inteligencia emocional en el Crecimiento Psicológico Padrón López, Gladys Aracelys y Sánchez de Gallardo, Marhilde

Tabla 2

Estadísticos descriptivos elementos de la motivación al logro, inteligencia emocional y Crecimiento Psicológico

\begin{tabular}{|c|c|c|c|c|c|}
\hline Variable & Dimensión & Indicador & Porcentaje & Interpretación & Dispersión \\
\hline \multirow{7}{*}{$\begin{array}{c}\text { Motivación } \\
\text { al Logro }\end{array}$} & \multirow{4}{*}{$\begin{array}{c}\text { Interior o intra } \\
\text { personal }\end{array}$} & Experticia & $95.7 \%$ & Muy Alta & Baja (3.07) \\
\hline & & Eficiencia & $94.9 \%$ & Muy Alta & Baja (4) \\
\hline & & Excelencia & $94 \%$ & Muy Alta & Baja (3.07) \\
\hline & & & $95.7 \%$ & Muy Alta & Baja (9.43) \\
\hline & \multirow[t]{3}{*}{$\begin{array}{l}\text { Dimensión } \\
\text { Exterior }\end{array}$} & $\begin{array}{l}\text { Impacto sobre el } \\
\text { crecimiento de } \\
\text { otras personas }\end{array}$ & $88 \%$ & Muy Alta & $\begin{array}{c}\text { Moderada } \\
(3.93)\end{array}$ \\
\hline & & $\begin{array}{l}\text { Calidad de } \\
\text { vida social }\end{array}$ & $86.3 \%$ & Muy Alta & $\begin{array}{c}\text { Moderada } \\
(3.82)\end{array}$ \\
\hline & & & $89.7 \%$ & Muy Alta & $\begin{array}{c}\text { Moderada } \\
(7.33)\end{array}$ \\
\hline \multirow[t]{6}{*}{$\begin{array}{l}\text { Inteligencia } \\
\text { Emocional }\end{array}$} & \multirow[t]{3}{*}{$\begin{array}{c}\text { Competencia } \\
\text { Personal }\end{array}$} & $\begin{array}{l}\text { Conciencia } \\
\text { de sí mismo }\end{array}$ & $76.9 \%$ & Muy Alta & $\begin{array}{c}\text { Moderada } \\
(7.02)\end{array}$ \\
\hline & & Autogestión & $88.9 \%$ & Muy Alta & $\begin{array}{c}\text { Moderada } \\
(11.58)\end{array}$ \\
\hline & & & $89.7 \%$ & Muy Alta & $\begin{array}{c}\text { Moderada } \\
(17.59)\end{array}$ \\
\hline & \multirow[t]{3}{*}{$\begin{array}{c}\text { Competencia } \\
\text { Social }\end{array}$} & $\begin{array}{c}\text { Conciencia } \\
\text { social }\end{array}$ & $86.3 \%$ & Muy Alta & $\begin{array}{c}\text { Moderada } \\
(8.97) \\
\end{array}$ \\
\hline & & $\begin{array}{c}\text { Gestión de } \\
\text { las relaciones }\end{array}$ & $93.2 \%$ & Muy Alta & $\begin{array}{c}\text { Baja } \\
(13.76)\end{array}$ \\
\hline & & & $89.7 \%$ & Muy Alta & Baja (21.8) \\
\hline \multirow[t]{4}{*}{$\begin{array}{l}\text { Crecimiento } \\
\text { Psicológico }\end{array}$} & & $\begin{array}{c}\text { Manejo positivo } \\
\text { del fracaso }\end{array}$ & $88 \%$ & Muy Alta & $\begin{array}{c}\text { Moderada } \\
(4.6)\end{array}$ \\
\hline & & $\begin{array}{l}\text { Manejo de la } \\
\text { incertidumbre }\end{array}$ & $89.7 \%$ & Muy Alta & $\begin{array}{c}\text { Moderada } \\
(4.05)\end{array}$ \\
\hline & & $\begin{array}{c}\text { Apertura al } \\
\text { cambio }\end{array}$ & $\begin{array}{l}50.4 \% \\
43.6 \% \\
\end{array}$ & $\begin{array}{c}\text { Alta } \\
\text { Muy Alta }\end{array}$ & Alta (2.32) \\
\hline & & Flexibilidad & $89.7 \%$ & Muy Alta & Baja (2.29) \\
\hline
\end{tabular}

Fuente: Padrón y Sánchez de Gallardo (2009) 
ubicó en un nivel muy alto, comportándose más homogénea por cuanto la dispersión en el grupo en estudio fue baja.

Crecimiento Psicológico: Elementos

Los resultados obtenidos indican que el grupo en estudio se ubicó en un nivel muy alto en relación al manejo positivo del fracaso (88\%) y manejo de la incertidumbre $(89.7 \%)$, evidenciándose una moderada dispersión.

En cuanto a la apertura al cambio, el grupo predominante se ubicó en un nivel alto $(50.4 \%)$, con una tendencia importante en el muy alto (43.6\%), lo cual se confirma con una moderada dispersión.

Los resultados en torno a la flexibilidad, ubican al grupo objeto de estudio en un nivel muy alto $(89.7 \%)$ con una baja dispersión, indicando la uniformidad de los mismos.

Resultados que reafirman los hallazgos encontrados por Padrón y Polanco (2006) en su artículo titulado "Crecimiento psicológico y motivaciones sociales: una aproximación prospectiva en estudiantes universitarios", donde se evidenció que de los tres motivos sociales (logro, poder y afiliación), es la motivación al logro la que tiene una correlación más elevada con el crecimiento psicológico explicando el $18 \%$ de la varianza en esta variable, confirmando a su vez los planteamientos del modelo expuesto por Romero (2000) al respecto.

\section{Conclusiones}

Considerando los resultados obtenidos y el modelo nombrado anteriormente, es posible afirmar que la competencia personal y social demostrada por el grupo en un nivel muy alto, se relaciona con la motivación al logro impulsando el crecimiento psicológico.

Podría esperarse entonces, que los sujetos objeto de estudio, al percibir la incertidumbre o el fracaso como un reto u oportunidad, se mantengan activados, procesando la información del entorno con flexibilidad, sin prejuicios, permitiendo enriquecer los conocimientos, al estar consciente de sus aprendizajes, y en consecuencia aprenden y crecen.

Las evidencias estarían revelando la tendencia a elaborar adecuadamente sus percepciones ante los cambios con los que se enfrentan, esto implicaría aperturarse a la experiencia, transformándola en oportunidades de desarrollo emocional, familiar, social y productivo, pudiendo acceder a una forma más armoniosa y creativa de manejar la situación de cambio; y desde la perspectiva expuesta por Romero (2000) contribuiría al crecimiento psicológico del individuo.

En tal sentido, el crecimiento psicológico, construcción integradora, entendida como interpretación de la realidad, permite que el individuo comprenda las limitaciones de su medio social, las supere y sea capaz de funcionar eficientemente, manejando la incertidumbre de forma abierta y creativa, comprendiendo de forma inteligente el fracaso tanto propio como el ajeno, siendo flexible en su construcción del mundo y de los eventos.

Lo anterior estaría demostrando la capacidad del grupo para generar construcciones alternativas de personas o situaciones que ayudan a crecer, significando de esa manera, la posibilidad de los individuos de incrementar su habilidad para construir de diversas formas un mismo evento, percibiendo distintas 
Motivación al logro e inteligencia emocional en el Crecimiento Psicológico Padrón López, Gladys Aracelys y Sánchez de Gallardo, Marhilde

perspectivas y asumiendo los cambios del entorno.

En cuanto a la motivación al logro, la dimensión interior, reveló que los sujetos exponen maestría sobre la tarea (experticia), rendimientos rápidos y económicos (eficacia), y resultados de calidad superior (excelencia). En términos de comportamiento, cualquiera de estos desempeños, o la combinación de los mismos, confieren al individuo cogniciones y afectos de un valor personal importante.

En tal sentido, los resultados inclinan a que el grupo en estudio, manifiesta compromiso en la búsqueda de nuevos aprendizajes, lo cual contribuye al desarrollo económico de la organización, comunidad o sociedad como un todo y hace referencia al impacto sobre el crecimiento de otros y la calidad de vida en general.

Esta información evidencia pensamientos y conductas orientadas al máximo desarrollo de sus potencialidades con la finalidad de obtener resultados de nivel superior, tanto para la satisfacción personal como para propiciar resultados óptimos que generen beneficios a otras personas.

De los planteamientos presentados puede señalarse, la asociación de este constructo motivacional con conductas de búsqueda de éxito, exigencia de niveles altos en la ejecución, persistencia en la realización de las tareas; pudiendo afirmarse que las personas con alta motivación de logro, como es el caso del grupo en estudio, desarrollan conductas relacionadas con la tendencia a esforzarse por conquistar el éxito en relación a un determinado nivel de excelencia.

Respecto a los elementos utilizados para su estudio, en función del mode- lo teórico expuesto por Goleman y otros (2004), los resultados indican que el grupo se caracteriza en su mayoría por tener un buen dominio y confianza de sí mismo, conocimiento de sus emociones, las cuales gestionan, controlándose, muestran honestidad e integridad, con flexibilidad ante los cambios.

Asimismo, establecen adecuadas relaciones con los demás, en forma empática, orientados al servicio, con conciencia de la organización en la cual están inmersos; gestionando sus relaciones en base al liderazgo, influencia, preocupación por el desarrollo de otros, establecimiento de vínculos, trabajo en equipo, colaboración, negociando para resolver los conflictos que puedan presentarse e iniciando o manejando el cambio.

En función de lo expuesto, puede establecerse un marco referencial que permita posteriormente orientar el diseño de estrategias, programas y planes específicos tanto a nivel individual como grupal, para reforzar y afianzar la motivación al logro y la inteligencia emocional, a objeto de promover el crecimiento psicológico en los individuos.

\section{Referencias Bibliográficas}

Cantoni Ibarra, Félix (2002). EI Factor Humano en la Organización, Colombia,Intermedio Editores, pp. 419.

Cooper, Robert y Sawaf, Ayman (1998). La Inteligencia Emocional Aplicada al Liderazgo y a las Organizaciones, Colombia Editorial Norma S.A, pp. 309.

Chiavenato, Idalberto (2002). Gestión del TaIento Humano, Colombia, McGraw Hill Interamericana, S.A. pp. 475. 
Chiavenato, Idalberto (2007). Administración de los recursos humanos, México, Mc Graw Hill Interamericana, pp. 500.

Dessler, Gary (2009). Administración de recursos humanos, México, Editorial Pearson Prentice Hall, pp 801.

DuBrin, Andrew (2008). Relaciones humanas. Comportamiento humano en el trabajo. México Edit. Pearson. Prentice Hall, pp. 358.

Feldman, Robert (2006). Psicología con aplicaciones en países de habla hispana. México Edit. Mc Graw Hill Interamericana, Pág. 592.

French, Wendell, Bell, Cecil, Zawacki, Robert (2007). Desarrollo Organizacional: Transformación y administración efectiva del cambio. México, Mc Graw Hill Interamericana, pp. 363.

Goleman, Daniel (2000). La Inteligencia Emocional en la Empresa, España, Javier Vergara Editor, pp. 460.

Goleman, Daniel, Boyatzis, Richard y Mckee, Annie (2004). El Líder Resonante Crea Más. El Poder de la Inteligencia Emocional, Editorial Melvin C. A. Venezuela, pp. 351.

Gómez Samper, Henry (2003). ¿El Fin de los Gurúes? Debates IESA, Volumen IX, numero 1, Diciembre, Venezuela, Editado por el Instituto de Estudios Superiores de Administración, pp. 6-6.

Ivancevich, John M.; Konopaske, Robert y Matteson, Michael T. (2006). Comportamiento organizacional. México: Editorial Mc Graw Hill Interamericana, pp. 260.

Larsen, Randy y Buss, David (2005). Psicología de la personalidad. Dominio del conocimiento sobre la naturaleza humana, México, .Editorial Mc. Graw Hill Interamericana, pp. 651.
Morris, Charles y Maisto, Albert (2005). Introducción a la Psicología. México: Editorial. Pearson Prentice Hall, pp 514.

Newstrom, John W. (2007). Comportamiento humano en el trabajo. México: Mc Graw Hill, pp. 501.

Padrón López, Gladys y Polanco, Edilsia (2006). Crecimiento Psicológico y Motivaciones Sociales: Una Aproximación Prospectiva en Estudiantes Universitarios. Revista Venezolana de Ciencias Sociales, Volumen 10, No. 1, Venezuela, Universidad Nacional Experimental Rafael María Baralt pp 236-252.

Reeve, Johnmarshall (2003). Motivación y Emoción, México, McGraw-Hill Interamericana Editores, S. A. de C. V, pp 623.

Ríos Cabrera, Pablo (2008). Psicología. La aventura de conocernos. Venezuela: Editorial Cognitus, pp 442.

Robbins, Stephen (2004). Comportamiento Organizacional. Conceptos, Controversias y Aplicaciones, México, Prentice Hall Hispanoamericana, S. A, pp 584.

Robbins, Stephen P. y Coulter, M. (2005). Administración. México: Editorial. Pearson Education, pp 614.

Romero García, Oswaldo (2000). Crecimiento Psicológico y Motivaciones Sociales, Venezuela, Ediciones ROGYA, pp. 172.

Sánchez de Gallardo, Marhilde y Linares Wilmara (2006). Autoestima y Gestión local de alcaldes. Revista de Artes y Humanidades UNICA. Año 7, No. 16, Venezuela, Universidad Católica Cecilio Acosta, pp 98-125.

Santrock John W. (2006). Psicología de la Educación, México, Editorial Mc. Graw Hill Interamericana, pp 554. 